\title{
Induction of xenobiotic-metabolizing enzymes in hepatocytes by beta-naphthoflavone: Time-dependent changes in activities, protein and mRNA levels
}

\author{
KATEŘINA LNĚNIČKOVÁ \\ LENKA SKÁLOVÁ \\ LUCIE STUCHLÍKOVÁ RAISOVÁ \\ BARBORA SZOTÁKOVÁ \\ PETRA MATOUŠKOVÁ* \\ Faculty of Pharmacy \\ Charles University \\ CZ-500 05 Hradec Králové \\ Czech Republic
}

Accepted November 2, 2017

Published online November 14, 2017

\begin{abstract}
In the present study, time-dependency of the induction effect of a selective inducer on the activity, protein and mRNA levels of cytochromes P450 1A1/2 (CYP1A1/2), $\mathrm{NAD}(\mathrm{P}) \mathrm{H}$ :quinone oxidoreductase 1 (NQO1) and glutathione S-transferases (GSTA), in primary culture of rat hepatocytes was tested and evaluated. To show the differences in responses of tested enzymes, the common aryl hydrocarbon receptor $(\mathrm{AhR})$ ligand agonist, beta-naphthoflavone (BNF), was used. Induction of CYP1A1/2 by BNF was detected at all time intervals and at all levels (i.e., mRNA, protein, enzyme activity). Different responses of NQO1 and GSTA upon BNF treatment were observed. Our results demonstrate that the responses of different xenobiotic-metabolizing enzymes to the inducer vary in time and depend on the measured parameter. For these reasons, an induction study featuring only one-time interval treatment and/ or one parameter testing could produce misleading information.
\end{abstract}

Keywords: aryl hydrocarbon receptor (AhR), beta-naphthoflavone, rat hepatocyte, time dependency, mRNA-protein correlation

Our bodies are exposed to many xenobiotics that can change the expression and/or activity of xenobiotic-metabolizing enzymes (XMEs), which, in turn, may affect the pharmacological and toxicological properties of administered drugs. Therefore, knowledge about the xenobiotics that modulate XME expression is very important. For this purpose, in vivo as well as in vitro studies might be used. Many studies have dealt with the induction effect of selected compounds on XMEs in the liver. However, most of these studies are limited only to a single time interval (e.g., 1, 2). Therefore, some induction effects could be missed if the interval from administration to analysis is too short or too long. The data obtained and their interpretation are very important, since the results from such assays determine whether drug-drug and/or herb-drug interaction studies need to be carried out.

\footnotetext{
*Correspondence; e-mail: matousp7@faf.cuni.cz
} 
K. Lněničková et al.: Induction of xenobiotic-metabolizing enzymes in hepatocytes by beta-naphthoflavone: Time-dependent changes in activities, protein and mRNA levels, Acta Pharm. 68 (2018) 75-85

The Food and Drug Administration (3) is about to recommend a single time interval for the assessment of enzyme induction to predict potential drug-drug interactions, but such a "one-point" approach can be misleading. Responses can vary at different time points and the results also depend on the method used for evaluation. In induction studies, the effects of tested substances for enzyme expression can be followed up using three parameters: enzyme activity, protein level and the corresponding mRNA level, with enzyme activity outcome considered the most relevant from pharmacological and/or toxicological points of view. However, the determination of enzyme activity is unpopular mainly because of material consumption and low specificity of the results. For these reasons, instead of measuring enzyme activity, measurements of mRNA and/or protein level are used more frequently (4).

When the induction effect of a tested xenobiotic for a certain enzyme is studied, great discrepancies often appear between the results obtained for activity, protein and/or mRNA measurements $(1,4-6)$. There are many potential reasons for low correlations, e.g., involvement of post-transcriptional regulations, direct influence of xenobiotics on enzyme activity, or a particular time-delay in response in terms of mRNA and protein level. In a one time-point study, the time delay regarding induced changes in mRNA and a corresponding protein seems to be most problematic. Such time-delays could vary among different enzymes and thus accurate results cannot be expected from a one-time interval induction study or from the measurement of only one or two parameters. To verify this hypothesis, we decided to measure induced changes in enzyme activities, protein and mRNA of selected hepatic xenobiotic-metabolizing enzymes at several time intervals.

In order to provide evidence of how different responses can occur upon treatment, we have focused on cytochromes P450 1A1/2 (CYP1A1/2), NAD(P)H:quinone dehydrogenase 1 (NQO1) and glutathione S-transferases A (GSTA), all of which are regulated via the aryl hydrocarbon receptor (AhR). AhR is a ligand-activated transcriptional factor that belongs to the bHLH-PAS (basic helix-loop-helix/Per-Arnt-Sim) family. AhR resides in the cytoplasm as an inactivated complex with chaperone proteins (HSP90, XAP2, p23). When the ligand binds to AhR, the whole complex is translocated to the nucleus, where the AhR nuclear translocator (ARNT) protein is bound to it. Chaperone proteins are released and the AhR/ARNT heterodimer binds to the xenobiotic responsive element (XRE) sequence and elevates the expression of target genes. Among the AhR target genes are genes for Phase I and II biotransformation enzymes (CYP1A1, CYP1A2, CYP1B1, NQO1, GSTs, UDPglucuronosyl transferases 1A1, 1A6, 1A9, 2B35) as well as genes involved in the regulation of development, proliferation and differentiation $(2,7)$. Further, a large number of co-regulators involved in the interaction between enhancers and promoters have been recognized (8). Typical AhR ligands are polycyclic aromatic hydrocarbons and halogenated aryl hydrocarbons along with numerous dietary plant constituents such as indole alkaloids (8).

The present study was designed to show the time-dependency of response to the inducer, the correlation of the tested parameters (mRNA level, protein level, enzyme activity) as well as differences between individual xenobiotic-metabolizing enzymes. For our study, we have selected $\beta$-naphthoflavone (BNF, 5,6-benzoflavone), a synthetic flavonoid that belongs to the exogenous ligands of $\mathrm{AhR}$ group and is often used as a positive control in the evaluation of CYP1A induction (9-11). To achieve our aim, we exposed primary rat hepatocytes to BNF and monitored the expression and activity of GSTA, NQO1 and CYP1A1/2 at several time intervals. 


\section{EXPERIMENTAL}

\section{Chemicals and reagents}

S-(5'-adenosyl)-L-methionine (SAM), L-glutathione (GSH), 1-chloro-2,4-dinitrobenzene (CDNB), menadione and coenzyme NAPDH were obtained from Sigma-Aldrich (USA). Fetal bovine serum and streptomycin sulfate were purchased from Invitrogen (USA). Bovine serum albumin (BSA) was obtained from Fluka (Switzerland). All other chemicals, HPLC or analytical grade, were obtained from Sigma-Aldrich.

\section{Ethical statement}

The animal protocols used in this work were evaluated and approved by the Ethics Committee of the Ministry of Education, Youth and Sports (Protocol MSMT-24185/2015-11). The protocols comply with the Guide for the Care and Use of Laboratory Animals (Protection of Animals from Cruelty Act No. 246/92, Czech Republic).

\section{Isolation and culture of rat hepatocytes}

Hepatocytes were isolated by a two-step collagenase method from female rat liver (Rattus norvegicus) (12). Isolated hepatocytes were rewashed three times and mixed with culture medium (1:1 mixture of Ham F12 and Williams' E, supplemented with $\mathrm{NaHCO}_{3}$ and penicillin/streptomycin). Viability of the hepatocytes was determined by a Trypan blue efflux test (accepted viability was $70-100 \%$ ).

$1 \times 10^{6}$ viable cells in $3 \mathrm{~mL}$ of culture medium were placed into a Petri dish $(6 \mathrm{~cm}$ in diameter) coated with collagen. Fetal calf serum was added to the culture medium (5\%) to enhance the attachment of cells. After $4 \mathrm{~h}$ of rest, the medium was changed to a medium containing $10 \mu \mathrm{mol} \mathrm{L}{ }^{-1} \mathrm{BNF}$ or $0.1 \%$ DMSO as a control. Cultures were maintained at $37^{\circ} \mathrm{C}$ in a humid air atmosphere with $5 \% \mathrm{CO}_{2}$ for up to $2,4,12$ and $24 \mathrm{~h}$.

\section{Preparation of subcellular fractions}

Cytosolic and microsomal fractions were obtained from the control and influenced hepatocytes suspended in $0.1 \mathrm{~mol} \mathrm{~L}^{-1}$ sodium phosphate buffer ( $\mathrm{pH}$ 7.4). The cells were homogenized using sonication with Sonopuls (Germany). Subcellular fractions were isolated by differential centrifugation of the cell homogenate. Subcellular fractions were stored at $-80^{\circ} \mathrm{C}$. Protein concentrations in the subcellular fractions were assayed using the bicinchoninic acid assay according to the Sigma-Aldrich protocol.

\section{Enzyme assays}

Cytosolic glutathione S-transferase (GST) activity was assayed as previously reported

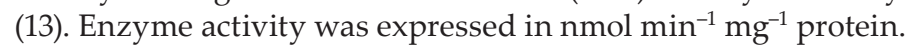

NQO1 activity was assayed spectrophotometrically by measuring cytochrome c reduction at $550 \mathrm{~nm}$ in the presence of NADH and menadione as described by Cullen et al. (14) in the cytosolic fraction. The NQO1 activity was calculated from the difference be- 
tween a non-inhibited and a dicumarol inhibited reaction. NQO1 activity was expressed in $\mathrm{nmol} \mathrm{min} \mathrm{mg}^{-1} \mathrm{mof}^{-1}$ protein.

The activity of ethoxyresorufin-O-deethylase (EROD, ascribed mainly to CYP1A1, partly to CYP1A2) was assayed in a microsomal fraction of treated or untreated hepatocytes using fixed wavelength fluorescence detection as described by Burke and Mayer (15), with the enzyme activity expressed in $\mathrm{pmol} \mathrm{min}^{-1} \mathrm{mg}^{-1}$ of protein.

\section{Western blotting}

Microsomal or cytosolic proteins ( $30 \mu \mathrm{g}$ in each lane) of hepatocytes were separated by SDS-PAGE and subsequently transferred onto nitrocellulose membranes $(0.45 \mu \mathrm{m})$ using a Trans-Blot ${ }^{\circledR}$ Turbo $^{\mathrm{TM}}$ Transfer System (Bio-Rad, USA). The membranes were blocked in $5 \%$ non-fat dry milk/TBS-Tween-20 for $2 \mathrm{~h}$. For the immunodetection of CYP1A1, CYP1A2, GSTA and NQO1, the membranes were probed overnight with primary antibodies [CYP1A1 - rabbit polyclonal, 1:1000 (Novus Biologicals, USA), CYP1A2 - mouse monoclonal, 1:1000 (Novus Biologicals), GSTA - goat polyclonal, 1:3000 (Abcam, UK), NQO1 - rabbit monoclonal, 1:3000 (Novus Biologicals)] diluted in TBS-Tween 20 supplemented with 1 $\%$ BSA, washed four times with TBS-Tween 20 buffer and probed with complementary secondary antibodies for $1 \mathrm{~h}$ [bovine anti-goat IgG-HRP, 1:3000, Santa Cruz Biotechnology (USA), bovine anti-mouse IgG-HRP, 1:10 000, Santa Cruz Biotechnology, bovine anti-rabbit IgG-HRP, 1:10 000, Santa Cruz Biotechnology]. The signal was detected using an enhanced Amersham ECL chemiluminescence kit (GE Healthcare Life Sciences, USA) according to the manufacturer's instructions. $\beta$-actin (mouse monoclonal, 1:3000, Abcam) served as the loading control. The intensity of bands was evaluated using a C-DiGit ${ }^{\mathrm{TM}}$ Blot Scanner (LICOR Biotechnology, USA). Protein levels were measured in two independent experiments.

\section{RNA isolation and quantitative real-time PCR}

Total RNA was isolated using TriReagent according to the manufacturer's instructions (Molecular Research Center, Inc., USA). RNA concentration and purity were determined spectrophotometrically. First strand cDNA was synthesized from $1 \mu \mathrm{g}$ total RNA using ProtoScript II reverse transcriptase (New England Biolabs, USA) and random hexamers. qPCR analyses were performed in a QuantStudio 6 Flex (Applied Biosystems, USA) using a qPCR Core kit for SYBR Green I (Eurogentec, Belgium) following the manufacturer's protocol. Primers were designed manually using Primer3 software (16) for CYP1A1 and 1A2 one primer set was designed to recognize both isoforms. Primer sequences and amplicon sizes are listed in Table I.

Calculations were based on the Delta-Delta Ct method (17), with data expressed as the fold change of treatment groups relative to the control. Beta-2 microglobulin (B2M) was used as the reference gene, with the gene's stability verified upon BNF treatment.

\section{Statistical analysis}

All calculations were done using Microsoft Excel and GraphPad Prism 6.04. Student's $t$-test was used for statistical evaluation of the differences between the treated groups and controls. $p$-value $<0.05$ was considered statistically significant. All measurements were performed in triplicate. 

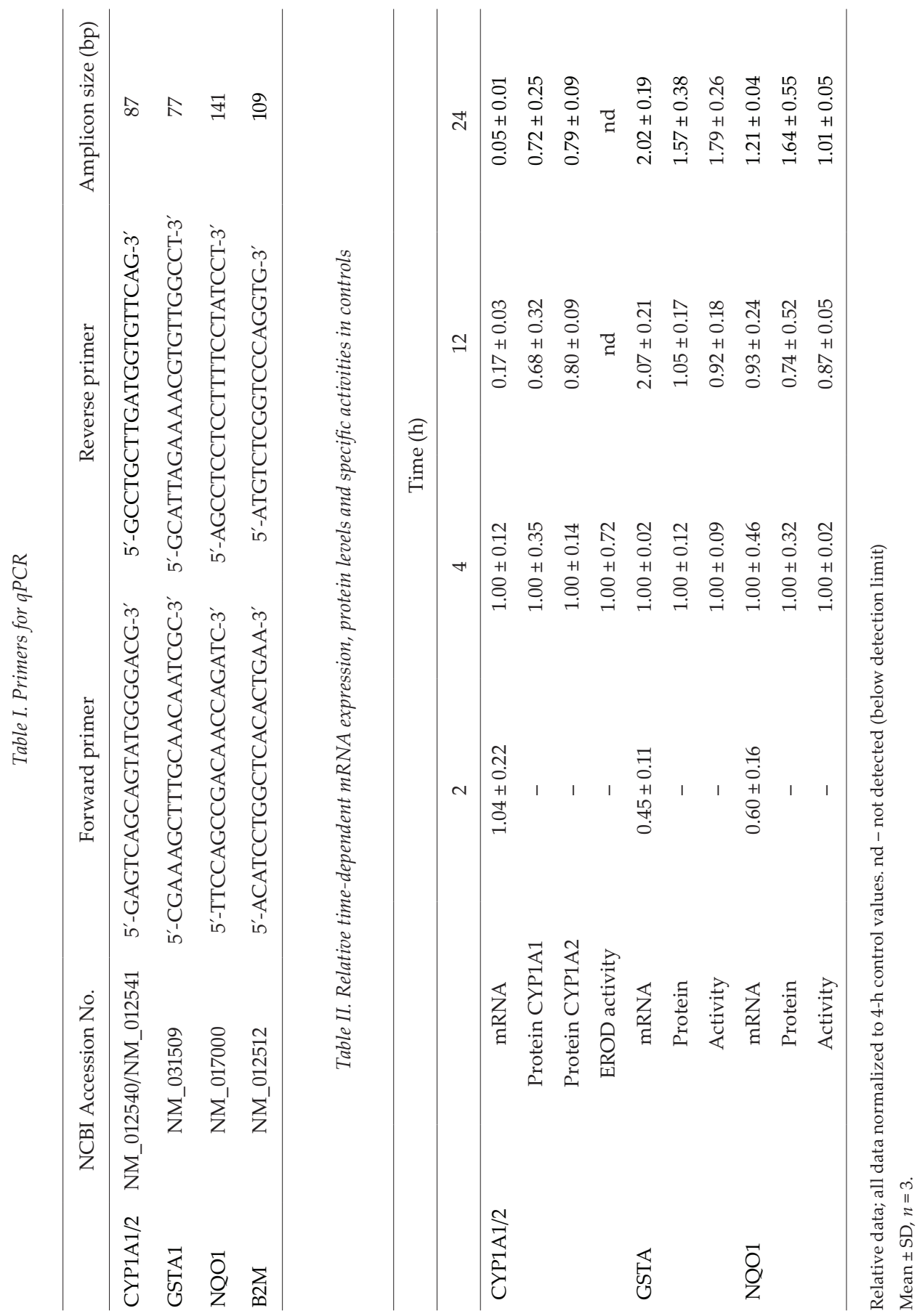


\section{RESULTS AND DISCUSSION}

\section{Time-dependent response of DMEs in the control}

In the present in vitro study, primary cultures of rat hepatocytes served as the model considered to be the most relevant for predicting in vivo induction $(4,18,19)$.

Firstly, we followed up the time-dependent changes of expression levels and activities of CYP1A1/2, NQO1 and GSTA in untreated hepatocytes during a 24 hour-cultivation (Table II). Great differences between individual enzymes as well as between other tested parameters were observed. Twenty-four hours after isolation, the level of CYP1A1/2 mRNA had decreased almost to zero, while NQO1 mRNA and GSTA mRNA increased up to 450 and $200 \%$, resp. Increase of NQO1 and GSTA expression could have been evoked by increased oxidation stress, since antioxidant-responsive elements (ARE) participate in the regulation of both enzymes $(20,21)$. Relative stability was found for the proteins. The amount of CYP1A1 and CYP1A2 proteins decreased only by $30 \%$, while the amount of NQO1 and GSTA proteins increased by approx. $60 \%$. The low mRNA-protein correlation implied the participation of post-transcriptional regulations in NQO1 expression. Surprisingly, the CYP1A1/2 activity had decreased to zero even 12 hours after the isolation of hepatocytes. The activity of GSTA remained unchanged during $24 \mathrm{~h}$, while NQO1 activity increased by $79 \%$ and correlated well with the increased protein level.

\section{The effect of BNF on DMEs}

CYP1A induction by BNF was clearly observed at all time intervals and at all levels. mRNA as well as the corresponding protein and enzyme activity increased gradually dur-

b)

a)
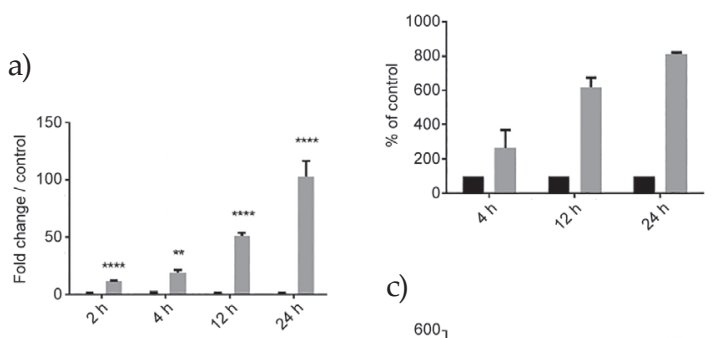

c)

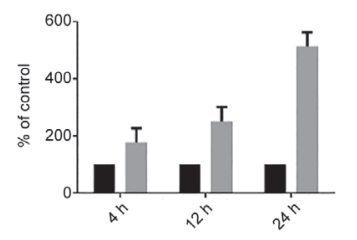

d)

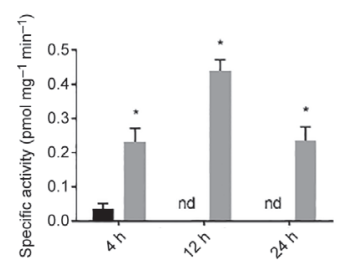

- Control BNF

Fig. 1. a) The mRNA expression (normalized to B2M), b) protein levels CYP1A1 and c) CYP1A2 (normalized to $\beta$-actin), and d) specific activity ( $\mathrm{pmol} \mathrm{min}^{-1} \mathrm{mg}^{-1}$ ) of CYP1A1/2 determined in the control and in BNF treated $\left(10 \mu \mathrm{mol} \mathrm{L}^{-1}\right)$ hepatocytes. The bars represent mean \pm SD after at least three independent measurements. Statistically significant difference $v$ s. control: ${ }^{*} p<0.05,{ }^{* *} p<0.01,{ }^{* * * *} p<$ 0.0001. BNF - beta-naphthoflavone, nd - not detected. 
a)

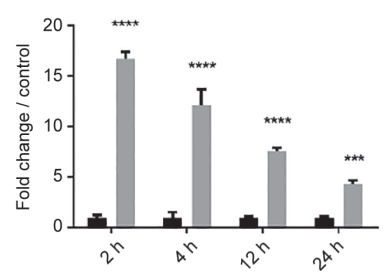

b)

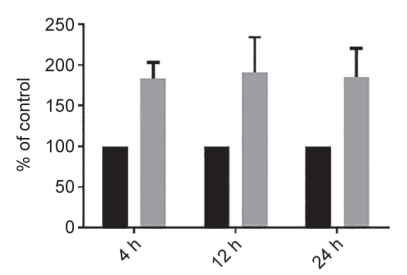

c)

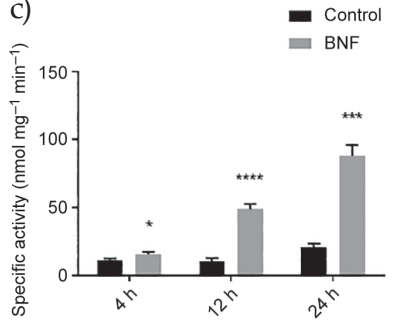

Fig. 2. a) The mRNA expression (normalized to B2M), b) protein levels (normalized to $\beta$-actin) and c) specific activity (nmol min $\mathrm{mg}^{-1}$ ) of NQO1 determined in the control and in BNF treated hepatocytes. The bars represent mean \pm SD after at least three independent measurements. Statistically significant difference vs. control: ${ }^{*} p<0.05,{ }^{* * *} p<0.001,{ }^{* * * *} p<0.0001$. BNF - beta-naphthoflavone.

a)

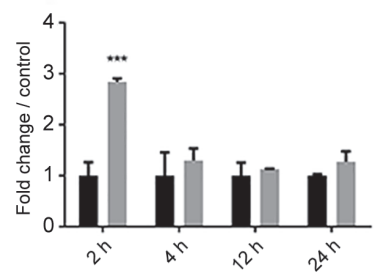

b)

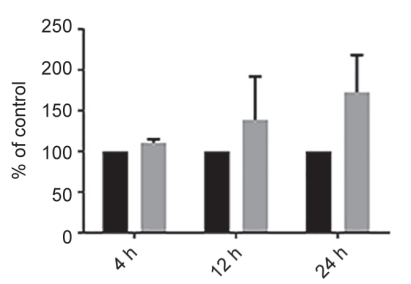

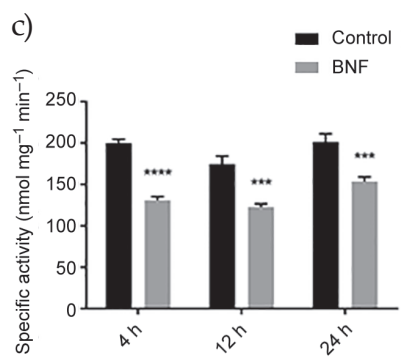

Fig. 3. a) The mRNA expression (normalized to B2M), b) protein levels (normalized to $\beta$-actin) and c) specific activity (nmol min-1 $\mathrm{mg}^{-1}$ ) of GSTA determined in the control and in BNF treated hepatocytes. The bars represent mean \pm SD after at least three independent measurements. Statistically significant difference $v$ s. control: ${ }^{* * *} p<0.001,{ }^{* * * *} p<0.0001$. BNF - beta-naphthoflavone.

ing a 24-h exposure of hepatocytes to BNF and all tested parameters correlated well. The response to $\mathrm{BNF}$ in hepatocytes was in agreement with the observation that the induction of hepatic CYP1A1/CYP1A2 mRNA and CYP1A activity in rats in vivo occurred within $2 \mathrm{~h}$ after BNF administration (22). A good correlation between the induction of CYP1A1/2 enzyme activities and mRNA expression in human hepatocytes has been also reported (4). For example, the plant flavonoid rutin induced the expression of CYP1A1 in a human liver cancer cell line even after a 48-h treatment (23). These results explain why CYP1A inducers are so easily revealed; any time-interval and any parameter is suitable for a CYP1A induction study.

On the other hand, NQO1 induction by BNF showed quite different timing, with expression of mRNA increasing rapidly, reaching a maximum level (16-fold of control) 2 hours after BNF administration. With increasing duration of exposure, the rate of induction diminished gradually, with only a 5-fold increase in the mRNA level found after 24-h exposure. The amount of NQO1 protein showed an increase after 4 hours, but only up to $180 \%$ of the control and the level of the protein did not change over the following $20 \mathrm{~h}$. Surprisingly, NQO1 activity did not correlate with the amount of NQO1 protein, since it rose gradually and reached a 3-fold control level after a 24-h exposure. From the observed results, we assume that after two hours the NQO1 mRNA level after BNF administration 
arose to a sufficient amount for higher protein production, which was indeed detected after 4-h. The increasing activity throughout our experiment without the corresponding protein level increase is hard to explain.

Regulation of GSTs is more complicated, with several mechanisms described (24). Nevertheless, the presence of XRE elements in the promotor region of many GSTs (namely, the GSTA family) was shown and the increase of hepatic GSTA after the administration of BNF to rats or mice was reported $(8,24,25)$. However, in our study, BNF increased GSTA mRNA only after a 2-h exposure and GSTA protein only after a 24-h exposure, which is a surprisingly long time span between mRNA and protein response. At all other timepoints, the mRNA level as well as protein level seemed to be unaffected. Moreover, GST activity decreased after BNF exposure at all time intervals, probably due to BNF-mediated enzyme inhibition. Flavonoids are potent GST inhibitors (26) and the inhibitory effect of residual test compounds in the hepatocyte monolayer can influence the results of activity measurement (9).

All the above-mentioned results clearly demonstrate that using only a one-time point induction study can render misleading information. When expressions of AhR dependent genes after a 24 -h treatment with $100 \mu \mathrm{mol} \mathrm{L}^{-1} \mathrm{BNF}$ were compared in various human laryngeal squamous carcinoma cell lines and in the hepatic carcinoma cell line, a massive increase of CYP1A1/2 mRNA and a slight or no increase of NQO1 mRNA were observed (27). If shorter treatments were used, the NQO1 mRNA increase would be more pronounced, and vice versa, namely, when levels of NQO1 and CYP1A1 mRNA in rat liver were measured following BNF i.p. administration, a significant increase of NQO1 mRNA (10fold) and CYP1A1 mRNA (800-fold) was observed after an 8-h treatment. However, after a 24-h treatment, NQO1 mRNA levels had not changed significantly (28). If only a 24-hour treatment was applied, NQO1 induction was not detected. In our study, only a 2-hour exposure of hepatocytes to BNF led to a significant increase in the mRNA of all tested enzymes. When longer exposure is applied, there is a growing risk of degradation of the induced mRNA.

In general, the correlation between mRNA and protein abundance is strongly affected by many factors and a high correlation cannot be assumed (29). Comparing the tested parameters, the quantification of mRNA is the most specific and precise parameter, but it can serve only for the evaluation of a transcriptional response to the tested compounds and has only a low predictive value from a pharmacological and/or toxicological point of view. On the other hand, enzyme activity testing has a greater potential to reveal drugdrug interaction, but it is much less specific.

\section{CONCLUSIONS}

Taken together, when the effect of the potential inducer is studied only at a one-time point, the induction effect can be underestimated and some valuable information can be lost. Our results showed that only some enzymes (e.g., CYP1A1/2) have a good correlation between enzyme activity, protein and mRNA level in response to inducers. In conclusion, protein quantification seems to bring the most valuable results, as it has good specificity and protein levels are relatively stable. Certainly, concomitant testing of at least two parameters is better for a more accurate interpretation of the induction study results. 
K. Lněničková et al.: Induction of xenobiotic-metabolizing enzymes in hepatocytes by beta-naphthoflavone: Time-dependent changes in activities, protein and mRNA levels, Acta Pharm. 68 (2018) 75-85.

Abbreviations/acronyms. - AhR - aryl hydrocarbon receptor, ARNT - AhR nuclear translocator, B2M - beta-2 microglobulin, BNF - beta-naphthoflavone, CYP - cytochrome P450, EROD - ethoxyresorufin-O-deethylase, GSTA - glutathione S-transferase alpha, HRP - horseradish peroxidase, NQO1 - NAD(P)H:quinone oxidoreductase 1, XME - xenobiotic-metabolizing enzymes, XRE - xenobiotics responsive element

Acknowledgements. - This work was supported by the Czech Science Foundation (Grant No. P303/12/G163) and by Charles University (Research Project SVV 260 416).

\section{REFERENCES}

1. H. Fujimura, N. Murakami, S. Miwa, C. Aruga and W. Toriumi, The suitability of rat hepatoma cell line H4IIE for evaluating the potentials of compounds to induce CYP3A23 expression, Exp. Toxicol. Pathol. 64 (2012) 527-533; https://doi.org/10.1016/j.etp.2010.11.010

2. D. B. Buckley and C. D. Klaassen, Induction of mouse UDP-glucuronosyltransferase mRNA expression in liver and intestine by activators of aryl-hydrocarbon receptor, constitutive androstane receptor, pregnane $X$ receptor, peroxisome proliferator-activated receptor alpha, and nuclear factor erythroid 2-related factor 2, Drug Metab. Dispos. 37 (2009) 847-856; https://doi.org/10.1124/ dmd.108.024190

3. Food and Drug Administration, Guidance for Industry: Drug Interaction Studies - Study Design, Data Analysis, and Implications for Dosing and Labeling, Draft Guidance, FDA, Center for Drug Evaluation and Research, Rockville 2006; https:/www.fda.gov/OHRMS/DOCKETS/98fr/06d-0344-gd10001. pdf; last access date October 3, 2017

4. L. Richert, G. Tuschl, C. Abadie, N. Blanchard, D. Pekthong, G. Mantion, J. C. Wr andebe S. O. Mueller, Use of mRNA expression to detect the induction of drug metabolising enzymes in rat and human hepatocytes, Toxicol. Appl. Pharmacol. 235 (2009) 86-96; https://doi.org/10.1016/j. taap.2008.1 J. Skorkovská 1.019, V. Hanušová, V. Tománková, E. Anzenbacherová, B. Lišková, P. Anzenbacher

5. P. Matoušková, H. Bártíková, I. Boušová, B. Szotáková, J. Martin, and L. Skálová, Effect of defined green tea extract in various dosage schemes on drug-metabolizing enzymes in mice in vivo, J. Funct. Foods 10 (2014) 327-335; https://doi.org/10.1016/j.jff.2014.06.026

6. O. H. Elshenawy and A. O. El-Kadi, Modulation of aryl hydrocarbon receptor regulated genes by acute administration of trimethylarsine oxide in the lung, kidney and heart of C57BL/6 mice, Xenobiotica 45 (2015) 930-943; https://doi.org/10.3109/00498254.2015.1032385

7. O. Hankinson, The aryl hydrocarbon receptor complex, Annu. Rev. Pharmacol. 35 (1995) 307-340; https://doi.org/10.1146/annurev.pa.35.040195.001515

8. H. M. Korashy and A. O. El-Kadi, The role of aryl hydrocarbon receptor in the pathogenesis of cardiovascular diseases, Drug Metab. Rev. 38 (2006) 411-450; https://doi. org/10.1080/03602530600632063

9. C. Abadie-Viollon, H. Martin, N. Blanchard, D. Pekthong, P. Bachellier, G. Mantion, B. Heyd, F. Schuler, P. Coassolo, E. Alexandre and L. Richert, Follow-up to the pre-validation of a harmonised protocol for assessment of CYP induction responses in freshly isolated and cryopreserved human hepatocytes with respect to culture format, treatment, positive reference inducers and incubation conditions, Toxicol. In Vitro 24 (2010) 346-356; https://doi.org/10.1016/j.tiv.2009.05.021

10. R. Glockner, A. Lieder and A. Lupp, Determination of CYP activity in precision-cut liver slices: whether to use intact slices or slice homogenate, Anal. Bioanal. Chem. 392 (2008) 1167-1172; https:// doi.org/10.1007/s00216-008-2238-y

11. M. Monshouwer, G. A. Van't Klooster, S. M. Nijmeijer, R. F. Witkamp and A. S. van Miert, Characterization of cytochrome P450 isoenzymes in primary cultures of pig hepatocytes, Toxicol. In Vitro 12 (1998) 715-723. 
K. Lněničková et al.: Induction of xenobiotic-metabolizing enzymes in hepatocytes by beta-naphthoflavone: Time-dependent changes in activities, protein and mRNA levels, Acta Pharm. 68 (2018) 75-85.

12. M. N. Berry, G. J. Barritt and A. M. Edwards, Isolated Hepatocytes: Preparation, Properties and Application, Elsevier, Amsterdam 1991, Vol. 21.

13. K. Lnenickova, E. Prochazkova, L. Skalova, P. Matouskova, H. Bartikova, P. Soucek and B. Szotakova, Catechins variously affect activities of conjugation enzymes in proliferating and differentiated Caco-2 cells, Molecules 21 (2016) e1186 (11 pages); https://doi.org/10.3390/molecules21091186

14. J. J. Cullen, M. M. Hinkhouse, M. Grady, A. W. Gaut, J. Liu, Y. P. Zhang, C. J. Weydert, F. E. Domann and L. W. Oberley, Dicumarol inhibition of NADPH:quinone oxidoreductase induces growth inhibition of pancreatic cancer via a superoxide-mediated mechanism, Cancer Res. 63 (2003) 55135520.

15. M. D. Burke and R. T. Mayer, Ethoxyresorufin: direct fluorimetric assay of a microsomal O-dealkylation which is preferentially inducible by 3-methylcholanthrene, Drug Metab. Dispos. 2 (1974) 583-588.

16. A. Untergasser, I. Cutcutache, T. Koressaar, J. Ye, B. C. Faircloth, M. Remm and S. G. Rozen, Primer3 - new capabilities and interfaces, Nucleic Acids Res. 40 (2012) e115 (12 pages); https://doi. org/10.1093/nar/gks596

17. K. J. Livak and T. D. Schmittgen, Analysis of relative gene expression data using real-time quantitative PCR and the 2(-Delta Delta C(T)) method, Methods 25 (2001) 402-408; https://doi.org/10.1006/ meth.2001.1262

18. Z. Dvorak, P. Maurel, M. J. Vilarem, J. Ulrichova and M. Modriansky, Expression and transcriptional activities of nuclear receptors involved in regulation of drug-metabolizing enzymes are not altered by colchiceine: Focus on PXR, CAR, and GR in primary human hepatocytes, Cell Biol. Toxicol. 23 (2007) 63-73; https://doi.org/10.1007/s10565-006-0127-8

19. M. Nishimura, A. Koeda, E. Suzuki, T. Shimizu, Y. Kawano, M. Nakayama and T. Satoh, Effects of prototypical drug-metabolizing enzyme inducers on mRNA expression of housekeeping genes in primary cultures of human and rat hepatocytes, Biochem. Bioph. Res. Commun. 346 (2006) 10331039; https://doi.org/10.1016/j.bbrc.2006.06.012

20. Y. Korenori, S. Tanigawa, T. Kumamoto, S. Qin, Y. Daikoku, K. Miyamori, M. Nagai and D. X. Hou, Modulation of Nrf2/Keap1 system by Wasabi 6-methylthiohexyl isothiocyanate in ARE-mediated NQO1 expression, Mol. Nutr. Food Res. 57 (2013) 854-864; https://doi.org/10.1002/mnfr.201200689

21. I. Bousova and L. Skalova, Inhibition and induction of glutathione S-transferases by flavonoids: possible pharmacological and toxicological consequences, Drug Metab. Rev. 44 (2012) 267-286; https://doi.org/10.3109/03602532.2012.713969

22. E. P. Chen, L. F. Chen, Y. Ji, G. Y. Tai, Y. H. Wen and H. Ellens, A mechanism-based mathematical model of aryl hydrocarbon receptor-mediated CYP1A induction in rats using beta-naphthoflavone as a tool compound, Drug Metab. Dispos. 38 (2010) 2278-2285; https://doi.org/10.1124/ dmd.110.034421

23. S. Karakurt, Modulatory effects of rutin on the expression of cytochrome P450s and antioxidant enzymes in human hepatoma cells, Acta Pharm. 66 (2016) 491-502; https://doi.org/10.1515/acph2016-0046

24. L. G. Higgins and J. D. Hayes, Mechanisms of induction of cytosolic and microsomal glutathione transferase (GST) genes by xenobiotics and pro-inflammatory agents, Drug Metab. Rev. 43 (2011) 92-137; https://doi.org/10.3109/03602532.2011.567391

25. O. H. Elshenawy and A. O. S. El-Kadi, Modulation of aryl hydrocarbon receptor-regulated enzymes by trimethylarsine oxide in C57BL/6 mice: In vivo and in vitro studies, Toxicol. Lett. 238 (2015) 17-31; https://doi.org/10.1016/j.toxlet.2015.06.1646

26. I. Bousova, J. Hajek, J. Drsata and L. Skalova, Naturally occurring flavonoids as inhibitors of purified cytosolic glutathione S-transferase, Xenobiotica 42 (2012) 872-879; https://doi.org/10.3109/0049 8254.2012.670737 
27. D. Brauze, K. Fijalkiewicz, M. Szaumkessel, K. Kiwerska, K. Bednarek, M. Rydzanicz, J. Richter, R. Grenman and M. Jarmuz-Szymczak, Diversified expression of aryl hydrocarbon receptor dependent genes in human laryngeal squamous cell carcinoma cell lines treated with beta-naphthoflavone, Toxicol. Lett. 231 (2014) 99-107; https://doi.org/10.1016/j.toxlet.2014.09.005

28. D. Brauze, M. Widerak, J. Cwykiel, K. Szyfter and W. Baer-Dubowska, The effect of aryl hydrocarbon receptor ligands on the expression of AhR, AhRR, ARNT, Hif1 alpha, CYP1A1 and NQO1 genes in rat liver, Toxicol. Lett. 167 (2006) 212-220; https://doi.org/10.1016/j.toxlet.2006.09.010

29. T. Maier, M. Guell and L. Serrano, Correlation of mRNA and protein in complex biological samples, FEBS Lett. 583 (2009) 3966-3973; https://doi.org/10.1016/j.febslet.2009.10.036 